\title{
Calidad de agua del Arroyo Guazú del Departamento Central evaluada mediante indicadores biológicos, parámetros fisicoquímicos y ecotoxicológicos
}

\author{
Water quality of the Guazú Stream in the Central Department \\ evaluated by means of biological indicators, physicochemical \\ and ecotoxicological parameters
}

\begin{abstract}
Tomás Rodrigo López Arias ${ }^{1 *} \odot$, Gustavo Villalba Duré ${ }^{2} \oplus$, Matias Daniel Policani Barrios ${ }^{1} \oplus$, Bárbara Camila Bogarín Cantero ${ }^{1} \odot$, Angelo Elli Mendoza ${ }^{1}{ }^{1}{ }^{1}$ Universidad Nacional de Asunción. Facultad de Ciencias Exactas y Naturales. Departamento de Biotecnología. Laboratorio de Biotecnología Ambiental. San Lorenzo, Paraguay. ${ }^{2}$ Universidad Nacional de Asunción. Centro Multidisciplinario de Investigaciones Tecnológicas. San Lorenzo, Paraguay. *Autor de correspondencia: tlopez@ @acen.una.py.
\end{abstract}

Recibido en versión modificada: 3 junio 2021

López Arias, T. R., Villalba Duré, G., Policani Barrios, M. D., Bogarín Cantero, B. C. \& Mendoza, A. E. (2021). Calidad de agua del Arroyo Guazú del Departamento Central evaluada mediante indicadores biológicos, parámetros fisicoquímicos y ecotoxicológicos.

Investigaciones y Estudios - UNA, 12(1), 4-18.

https://doi.org/10.47133/IEUNA2111

Resumen. Las aguas de arroyos urbanos del Área Metropolitana del Gran Asunción soportan una gran presión por la contaminación antrópica de origen urbano e industrial. El Arroyo Guazú es un cauce hídrico que atraviesa las Ciudades de Nemby y San Antonio y está afectado por la contaminación en todo su curso. La calidad de sus aguas con base a sus características fisicoquímicas, biológicas y ecotoxicológicas fue utilizada en este estudio para compararlo con la legislación nacional vigente. Se colectaron muestras de agua en cinco puntos diferentes en los meses de julio y noviembre del año 2017. Los resultados de los parámetros fisicoquímicos indican que sus aguas poseen características de Clase II y Clase III según lo establecido por el Ministerio de Ambiente y Desarrollo Sostenible de Paraguay para aguas superficiales. Algunos parámetros tales como nitrato, nitrógeno total, fósforo total, oxígeno disuelto, $\mathrm{DBO}_{5}$ y coliformes fecales presentaron valores fuera de los límites permitido para la Clase II. El Índice de Calidad de Agua (ICA) otorgó calificaciones "Regular" y "Mala", preferentemente hacia la cuenca baja en zona de su desembocadura al Río Paraguay, mientras que el Índice de Estado Trófico categorizó al arroyo entre mesotrófico a supereutrófico. En relación al zooplancton, se registró una disminución en la diversidad hacia la desembocadura. Por tanto, los resultados mostraron un aumento de la contaminación acuática. Los ensayos ecotoxicológicos de Daphnia magna y Lactuca sativa no detectaron presencia de 
contaminantes tóxicos en las aguas del Arroyo Guazú.

Palabras clave. contaminación, arroyos urbanos, coliformes.

Abstract. The waters of urban streams in the Metropolitan Area of Greater Asunción are under great pressure from anthropogenic pollution of urban and industrial origin. The Guazú Stream is a watercourse that crosses the cities of Nemby and San Antonio and is affected by pollution throughout its course. The quality of its water based on its physicochemical, biological and ecotoxicological characteristics was used in this study to compare it with current national legislation. Water samples were collected at five different points in the months of July and November 2017. The results of the physicochemical parameters indicate that its waters possess class II and class III characteristics as established by the Ministry of Environment and Sustainable Development of Paraguay. Some parameters such as nitrate, total nitrogen, total phosphorus, dissolved oxygen, BOD5 and fecal coliforms presented values outside the allowed limits. The Water Quality Index gave "Fair" and "Poor" ratings, preferably towards the lower basin in the area where it flows into the Paraguay River, while the trophic status index categorized the stream as mesotrophic to hypereutrophic. There was a decrease in zooplankton diversity towards the mouth of the river. Therefore, the results indicated an increase in aquatic pollution. Ecotoxicological tests on Daphnia magna and Lactuca sativa did not detect the presence of toxic pollutants in the waters of the Guazú Stream.

Keywords. contamination, urban streams, coliforms.

\section{INTRODUCCIÓN}

La Organización de las Naciones Unidas para la Alimentación y la Agricultura (FAO) en su reporte del 2015 indica que en Paraguay la contaminación por efluentes es una de las principales amenazas a los recursos hídricos. Si bien existen a nivel local estudios de monitoreos de calidad de aguas en las cuencas de los Río Paraguay, Paraná, Apa y otros, realizados por el Ministerio del Ambiente y Desarrollo Sostenible (MADES), las informaciones no se encuentran disponibles de forma sistematizada para un análisis más completo (FAO, 2015).

Además de presentar riesgos para la salud humana (Craggs, 2017), los contaminantes no monitoreados pueden introducirse y ascender en la cadena trófica o bioacumularse (Londoño Franco, L. et al., 2016). Por tal motivo, existe interés creciente en el estudio de la calidad de agua de los ecosistemas hídricos urbanos, ya que permiten observar las consecuencias del crecimiento poblacional desordenado y la falta de disposición adecuada de residuos sólidos y líquidos sobre las aguas superficiales y subterráneas. Por ejemplo, existen casos que reportan la presencia de coliformes totales, E. coli, y elevados niveles de nitratos en aguas de pozo de la Ciudad de San Lorenzo (Houben et al., 2012). Estos contaminantes pueden afectar la salud humana e incluso se corre el riesgo de que lleguen a los acuíferos.

Para evaluar la calidad de agua se recurre frecuentemente al empleo de índices de calidad de agua que son valores asignados producto de una combinación de parámetros fisicoquímicos, microbiológicos y su finalidad es asignar un valor para clasificarlo según su calidad o para su uso (Orozco Barrenetxea et al., 2003). Los valores de los 
parámetros de calidad de agua en Paraguay están estipulados en la resolución $\mathrm{N}^{\mathrm{o}} 222$ del MADES en la cual clasifica los cuerpos de agua según su uso y les asigna unos valores máximos permisibles. Sin embargo, existen otros métodos para evaluar la calidad de las aguas que no son utilizados por MADES, por ejemplo, a través de organismos bioindicadores o ensayos ecotoxicológicos que podrían complementar el análisis del cauce hídrico.

Los bioindicadores son organismos que, por sus características ecológicas, proporcionan información concreta sobre el estado ambiental de un ecosistema o sobre el efecto de algún contaminante sobre la biodiversidad acuática (Espino et al., 2000). El empleo de los bioindicadores no sólo permite diagnosticar la salud de un ecosistema acuático, sino que también permite evaluar el impacto sobre el ser humano, tanto a nivel sanitario como a nivel económico y medioambiental. Entre los bioindicadores más empleados se encuentran los macroinvertebrados (crustáceos, insectos y moluscos), y los microorganismos (bacterias, protozoarios, hongos y el plancton), cada uno con sus diferentes metodologías de estudio según los objetivos del trabajo y al tipo de ecosistema (Samarez et al., 2014). En el caso del zooplancton, su rápido crecimiento poblacional, la diversidad de biomasa, su elevada riqueza de grupos taxonómicos y su sensibilidad a los cambios ambientales y biológicos, le otorgan un elevado potencial como bioindicador (Parmar et al., 2016).

En lo referente a los parámetros ecotoxicológicos, Lactuca sativa (lechuga) y Daphnia magna (pulga de agua) son organismos indicadores comúnmente empleados para medir la toxicidad. Los ensayos de toxicidad aguda con $L$. sativa evalúan el efecto de un contaminante sobre la germinación de la semilla y el desarrollo de la plántula en su primera etapa de crecimiento. Estos ensayos permiten evaluar los posibles efectos fitotóxicos de las aguas y sedimentos. Por otro lado, las especies del género Daphnia son las más utilizadas como organismos de prueba o de referencia en pruebas de toxicidad debido a su facilidad de cultivo en el laboratorio, la reproducción partenogenética, y el corto ciclo de vida con la producción de un alto número de crías (Castillo Morales, 2004). D. magna es ampliamente utilizado para el monitoreo de impactos sobre ambientes acuáticos (Hodkinson \& Jackson, 2005) debido a su sensibilidad a agentes contaminantes. Al igual que $L$. sativa, que también ha demostrado sensibilidad a contaminantes ambientales en diferentes matrices como agua y sedimentos (Alonso \& López, 2015).

El Arroyo Guazú conforma la denominada cuenca Mbocayaty Guazú que forma parte del Acuífero Patiño, con un área de $47 \mathrm{~km}^{2}$, abarca parte de la Ciudad de Fernando de la Mora, Villa Elisa, Nemby y San Antonio. Comprende a dos cauces hídricos que se unen formando el mencionado arroyo que finalmente desemboca en el Río Paraguay (Monte Domecq \& Báez, 2007). Esta zona se caracteriza por los niveles freáticos elevados, la cual se constituyen en una problemática que dificulta la disposición de efluentes domiciliarios, generando pequeños cursos de aguas cloacales a cielo abierto que se agrava por el vertido de residuos sólidos sin control alguno (INCLAM, 2017). Asimismo, en la cuenca del Arroyo Guazú los pobladores permanentemente realizan denuncias relacionadas a los efluentes desechados por las 
industrias aledañas a la zona. Comúnmente las denuncias describen efluentes provenientes de servicios generales para el transporte, talleres de mecánica, pintura y chapa, industrias metalúrgicas, petroleras, de elaboración de bebidas, textiles y frigoríficos (las curtiembres y mataderos). Estos efluentes generan pestilencia y colorantes que imposibilitan su uso doméstico los cuales podrían representar un riesgo para la salud de los pobladores de la Ciudad de San Antonio (Ruiz Díaz, 2019). Por tanto, los efectos combinados de las actividades domésticas e industriales hacen que las aguas del Arroyo Guazú no puedan ser utilizadas para consumo o recreación a causa de la presencia de coliformes fecales y otros contaminantes (Otero, 2019).

A pesar de los potenciales riesgos y la importancia del análisis del Arroyo Guazú, hasta el momento existen pocos estudios publicados sobre la calidad de agua del arroyo y su aporte de contaminantes al Río Paraguay. Por tal motivo, el objetivo de este estudio es evaluar la calidad del agua del Arroyo Guazú mediante un análisis descriptivo del cumplimiento del padrón de calidad de aguas superficiales establecido por el MADES (MADES, 2002; MADES, 2006) así como índices de calidad de agua (ICA), estado trófico (IET), de diversidad zooplanctónica y ensayos ecotoxicológicos de modo a proporcionar información exhaustiva acerca del cauce hídrico y los posibles contaminantes presentes.

\section{MATERIALES Y MÉTODOS}

Sitio de estudio y estrategia de toma de muestras. La toma de muestras de agua fue realizada en dos campañas (entre julio y noviembre del 2017), por lo menos 10 días después de precipitaciones en la zona. Los sitios de muestreo fueron seleccionados teniendo en cuenta la accesibilidad y la localización de la cuenca (alta, media y baja). Así, el primer sitio denominado AG (UTM $x=447071,78 ; y=791492,19$ ), corresponde a la cuenca alta, ubicada en la Ciudad de Nemby, en inmediaciones de una fábrica productora de plásticos. El segundo sitio, P1 (UTM x=442635,82; $\mathrm{y}=7191109,72)$, corresponde al brazo derecho, y el tercero P2 (UTM x=443305,59, y=7191292,36) al brazo izquierdo, ambos se consideran como parte de la cuenca media. Los sitios ubicados en la cuenca baja son P3 (UTM x=442491,88; y=7190073,68), mientras que, P4 (UTM $\mathrm{x}=441487,51$; $\mathrm{y}=7189499,98$ ) es la desembocadura al Río Paraguay. Tanto P1, P2 y P3 son sitios ubicados en zonas altamente urbanizadas, donde se aprecia la disposición de residuos sólidos a modo de vertederos urbanos en la ribera del cauce hídrico (Figura 1). Por su parte, P4 tiene las características más propias de un delta, y según el nivel del Río Paraguay y los sedimentos transportados por el arroyo, el agua circula a menor velocidad. En esta zona también están ubicadas varias curtiembres que vierten sus desechos al cauce.

La colecta, conservación y transporte de muestras de agua fueron realizadas de manera manual siguiendo los procedimientos establecidos en el Standard Methods for the examination of water and waste-wasters (APHA/AWWA/WEF, 2012) para su posterior análisis. Se utilizaron frascos de polipropileno de $1 \mathrm{~L}$ para los ensayos toxicológicos y físicoquímicos, frascos de vidrio color ámbar para la determinación de fósforo y clorofila- $a$, y envases 
de plástico estériles para las determinaciones microbiológicas. Las muestras fueron conservadas a $4{ }^{\circ} \mathrm{C}$ hasta su traslado al laboratorio.

\section{Análisis fisicoquímicos y microbiológicos. Se} seleccionaron parámetros necesarios para calcular los índices ICA e IET (Brown et al., 1970; Carlson, 1977), además de aquellos requeridos para determinar la calidad del agua según lo establecido en la Resolución 222/02 del MADES. Las determinaciones se realizaron en el Laboratorio de Calidad de Agua (Facultad de Ciencias Exactas y Naturales/UNA), estas son: Demanda Bioquímica de Oxígeno a los cinco días $\left(\mathrm{DBO}_{5}\right)$, Demanda Química de Oxígeno (DQO), nitratos $\left(\mathrm{NO}_{3}{ }^{-}\right)$, nitrógeno total Kjeldahl (NTK), ortofosfato $\left(\mathrm{PO}_{4}^{-3}\right)$, fósforo total (PT), turbidez y clorofila- $a$. Los análisis se realizaron siguiendo las técnicas analíticas descritas en el Standard Methods for the examination of water

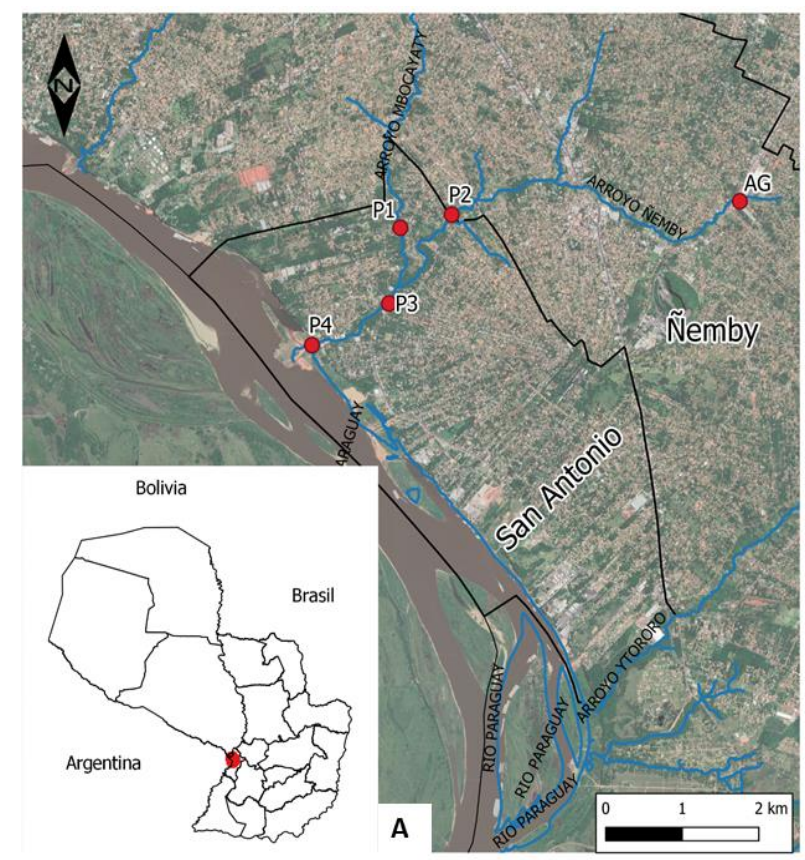

and waste-wasters (APHA/AWWA/WEF, 2012). Otros parámetros como el $\mathrm{pH}$, conductividad, oxígeno disuelto (OD) y sólidos disueltos totales (SDT) fueron determinados con un equipo multiparamétrico HANNA serie HI 9828. Por último, el recuento de coliformes fecales (CF) se realizó por el método de filtración de membrana (APHA/AWWA/WEF, 2012).

Análisis del zooplancton. Para la colecta del zooplancton, fueron realizados arrastres horizontales con una red de plancton de $68 \mu \mathrm{m}$ de abertura de malla. Posteriormente, las muestras concentradas fueron depositadas en botellas de plástico de $125 \mathrm{~mL}$ y fijadas con formol al $4 \%$ para preservar los organismos presentes. Posteriormente las muestras fueron analizadas en una caja de Petri, tomando submuestras de $10 \mathrm{~mL}$ con ayuda de un microscopio invertido.
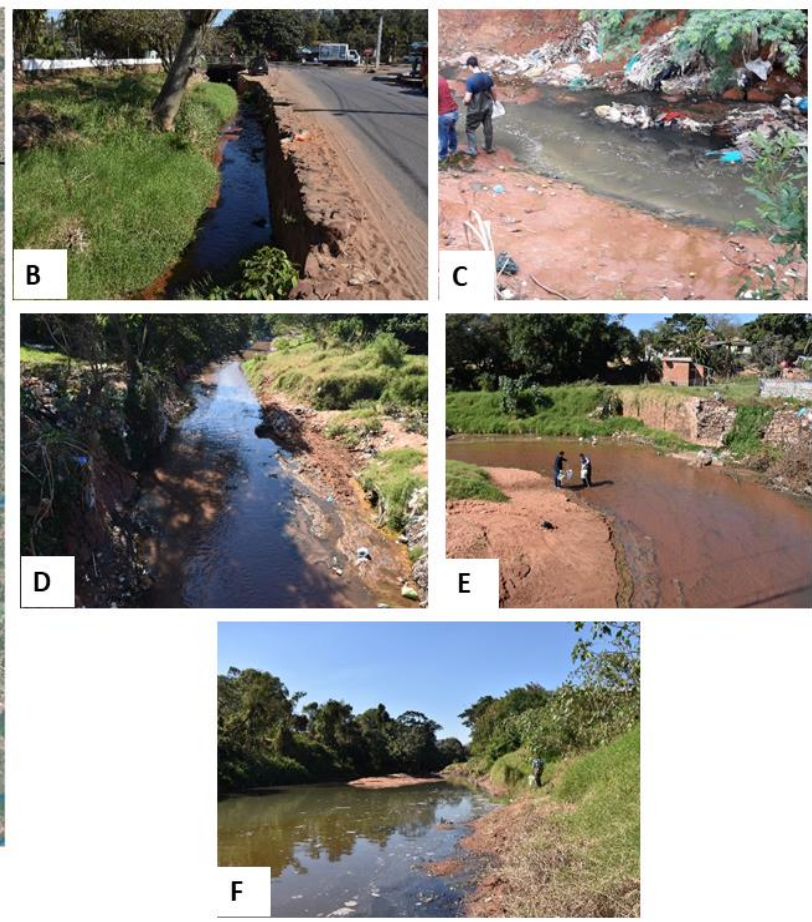

Figura 1. Sitios de estudio y colecta de muestras de agua del Arroyo Guazú entre julio y noviembre del 2017.

Referencias: (A) ubicación geográfica del área de estudio (B) Sitio AG. (C) sitio P1 (D) sitio P2 (E) sitio P3

(F) sitio P4. Fuente: (QGIS, 2020). 
Se examinaron tres grupos taxonómicos de zooplancton (rotíferos, cladóceros y copépodos) utilizando material bibliográfico especializado (Elmoor-Loureiro, 1997; Joko, 2011). Los organismos fueron identificados hasta nivel de género o especie de ser posible y posteriormente cuantificados.

Análisis de diversidad zooplanctónica. La diversidad zooplanctónica fue cuantificada mediante la estimación de la riqueza de taxones (S), es decir, el número de taxones presentes en las muestras, y el índice de Pielou (Magurran, 2005) para representar la equidad de la comunidad acuática.

\section{Ensayos ecotoxicológicos. Toxicidad aguda en}

Lactuca sativa L. Se utilizaron semillas de Lactuca sativa, obtenidas de un comercio local de agroproductos. Se trabajó con un control negativo, y concentraciones diferentes por cada muestra: el factor de dilución seleccionado corresponde a 0,5, así a partir de $100 \%$, se preparan otras al $50 \%$, $25 \%, 12,5 \%$ y $6,25 \%$ (tratamientos). Se utilizaron cápsulas de Petri de plástico descartables estériles, con papel filtro Qualy, de 14 micras de poro y 12,5 cm de diámetro. Los ensayos se realizaron según las recomendaciones de Castillo Morales (2004). Se determinó el porcentaje de inhibición (IP), entendida como el porcentaje de inhibición de la prolongación de la raíz expuesta al agua del arroyo, en relación al crecimiento en el control. El IP se obtiene según la ecuación 1:

\section{Ecuación 1}

$I P=\left(\frac{\text { Longitud de raices del tratamiento }- \text { longitud de raices del control }}{\text { longitur de raices del control }}\right) \times 100$

El criterio de punto final fue la concentración efectiva $50 \quad\left(\mathrm{CE}_{50}\right)$ a las 120 horas de exposición. Esto corresponde a la concentración que induce a la inhibición (IP) del crecimiento radicular en un $50 \%$ respecto al control.

Toxicidad aguda con Daphnia magna. El mantenimiento y las pruebas se realizaron siguiendo las recomendaciones de Castillo Morales (2004). Se utilizaron 10 neonatos de menos de 24 horas de nacidas provenientes del kit comercial Daphtoxkit $\mathrm{F}$ magna (MicroBioTests Inc., Bélgica), que fueron expuestos a diferentes concentraciones del agua del arroyo con un factor de dilución de 0,5. Como medio de dilución y el control negativo se utilizó agua dura reconstituida y como control positivo se utilizó dicromato de potasio $\left(\mathrm{K}_{2} \mathrm{Cr}_{2} \mathrm{O}_{7}\right)$ a una concentración de $2 \mathrm{mg} . \mathrm{L}^{-1}$. Los cladóceros fueron expuestos a $30 \mathrm{~mL}$ de cada tratamiento, los tratamientos se realizaron por triplicado. $\mathrm{La}$ temperatura de trabajo fue de $22 \pm 2{ }^{\circ} \mathrm{C}$ y el fotoperiodo de 16/8 horas de luz y oscuridad. Transcurrido las 48 horas de exposición se evaluó el número de organismos muertos por cada tratamiento, utilizando una lupa estereoscópica con aumento de 40x. El criterio de punto final fue la concentración letal $50\left(\mathrm{CL}_{50}\right)$ a las 48 horas de exposición.

Índice de Calidad de Agua (ICA). Para la determinación del Índice de Calidad del Agua (ICA) se utilizó la clasificación propuesta por Brown et al., (1970), con la fórmula de función ponderada multiplicativa obtenida mediante la ecuación 2.

Ecuación 2

$$
I C A=\prod_{i=1}^{9} \cdot\left(S u b_{i}{ }^{w_{i}}\right)
$$

Donde $\Pi$ representa la operación multiplicativa de las variables $\mathrm{Q}$ elevadas a la $\mathrm{W}$; $\mathrm{w}_{\mathrm{i}}$ : pesos relativos asignados a cada parámetro $\left(\mathrm{Sub}_{\mathrm{i}}\right)$; y $\mathrm{Sub}_{\mathrm{i}}$ : 
subíndice del parámetro i. Los parámetros utilizados para la determinación fueron: coliformes fecales, $\mathrm{pH}, \quad \mathrm{DBO}_{5}, \quad$ nitratos, ortofosfato, temperatura, turbidez, SDT y OD (\%).

Índice de Estado Trófico (IET). El estado trófico fue evaluado a través del cálculo del índice de estado trófico (IET) para los parámetros de clorofila- $a$ y fósforo total (Carlson, 1977), aplicando las fórmulas y clasificación recomendada por la Companhia Ambiental do Estado de São Paulo (CETESB, 2016) para ecosistemas lóticos (Ecuaciones 3, 4 y 5):

Ecuación 3

$\operatorname{IET}($ Clorofila $-a)=10 \times\left(6-\left(\frac{0.7-0.6 \ln C l a}{\ln 2}\right)\right)-20$
Ecuación 4

IET (Fósforo total)

$$
=10 \times\left(6-\left(\frac{0,42-0,36 \ln P T}{\ln 2}\right)\right)-20
$$

Ecuación 5

$$
\text { IET prom }=(I E T C l a+I E P T) / 2
$$

Donde lnCla es el logaritmo natural de la concentración de clorofila- $a$ en $\mu \mathrm{g} \cdot \mathrm{L}^{-1}$, $\operatorname{lnPT}$ es el logaritmo natural de la concentración de fósforo total en $\mu \mathrm{g} \cdot \mathrm{L}^{-1}$.

\section{RESULTADOS}

En la tabla 1 se presentan los resultados de los análisis fisicoquímicos y microbiológicos.

\begin{tabular}{|c|c|c|c|c|c|c|c|c|c|c|c|}
\hline Local & $\mathrm{AG}$ & & $\mathrm{P} 1$ & & $\mathrm{P} 2$ & & P3 & & $\mathrm{P} 4$ & & Ref* \\
\hline $\begin{array}{l}\text { Muestreo/ } \\
\text { Variables** }\end{array}$ & $1^{\circ}$ & $2^{\circ}$ & $1^{\circ}$ & $2^{\circ}$ & $1^{\circ}$ & $2^{\circ}$ & $1^{\circ}$ & $2^{\circ}$ & $1^{\circ}$ & $2^{\circ}$ & $\begin{array}{l}\text { MADES } \\
\text { Clase II }\end{array}$ \\
\hline $\mathrm{pH}$ & 7,47 & 6,25 & 7,33 & 7,00 & 7,49 & 6,63 & 7,43 & 6,84 & 7,45 & 7,04 & 6 a 9 \\
\hline Clor- $a$ & 1,60 & 9,08 & 0,53 & 2,14 & 1,14 & 4,45 & 10,95 & 3,20 & 0,33 & 7,34 & SR \\
\hline Turb. & 1,98 & 3,17 & 5,61 & 4,15 & 17,20 & 42,7 & 7,69 & 22,1 & 15,60 & 20,9 & $\leq 10$ \\
\hline $\mathrm{NO}_{3}^{-}$ & 14,99 & 0,18 & 1,06 & 0,424 & $<0,01$ & 0,02 & 0,86 & $<0,01$ & 0,65 & $<0,01$ & 10 \\
\hline NTK & 0,15 & 0,24 & 0,15 & 0,14 & 0,51 & 1,92 & 0,10 & 0,76 & 0,18 & 1,05 & 0,6 \\
\hline $\mathrm{PT}$ & 0,12 & 0,13 & 0,10 & 0,18 & 0,62 & 1,01 & 0,23 & 1,48 & 0,17 & 0,27 & 0,05 \\
\hline $\mathrm{PO}_{4}^{-3}$ & 0,08 & 0,10 & 0,06 & 0,15 & 0,30 & 0,69 & 0,17 & 0,20 & 0,11 & 0,23 & SR \\
\hline OD & 8,10 & 7,80 & 7,70 & 7,20 & 1,60 & 4,00 & 5,70 & 3,40 & 5,20 & 3,30 & $\geq 5$ \\
\hline $\mathrm{DBO}_{5}$ & 3,00 & 4,40 & 4,20 & 4,70 & 44,00 & 41,30 & 11,40 & 39,30 & 16,80 & 39,30 & $\leq 5$ \\
\hline DQO & 93,33 & 17,86 & 24,89 & 22,86 & 91,56 & 809,3 & 39,56 & 241,5 & 64,89 & 76,27 & SR \\
\hline SDT & 118 & 135 & 123 & 150 & 296 & 243 & 191 & 183 & 174 & 471 & $<500$ \\
\hline $\mathrm{CF}$ & 35 & 30 & 215 & 12 & 1.117 & 500 & 543 & 600 & 34 & 700 & $<1.000$ \\
\hline
\end{tabular}

Tabla 1. Parámetros fisicoquímicos de las aguas del arroyo Guazú por punto y por campaña.

* Referencia: Resolución $\mathrm{N}^{\circ}$ 222/02 MADES para aguas de Clase II.

**Observación: $\mathrm{pH}(\mathrm{UpH})$, Clor- $a$ (Clorofila- $a$, $\mu \mathrm{g} . \mathrm{L}^{-1}$ ), Turb. (Turbidez, NTU), $\mathrm{NO}_{3}^{-}$(Nitrato, mg.L $\mathrm{L}^{-1}$ ), NTK (Nitrógeno Total Kjeldahl mgN.L ${ }^{-1}$ ), PT (Fósforo total, mg.L $\mathrm{L}^{-1}$ ), $\mathrm{PO}_{4}^{-3}$ (Ortofosfato, mg.L $\mathrm{L}^{-1}$ ), OD (Oxígeno disuelto, mg.L $\mathrm{L}^{-1}$ ), DBO5 (Demanda bioquímica de

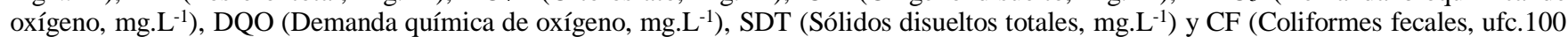
$\left.\mathrm{mL}^{-1}\right)$. 
Los resultados se comparan con los parámetros máximos establecidos en la Resolución No 222/02 del MADES para aguas de Clase II. El trabajo se enfoca en el cumplimiento de la normativa nacional de calidad de aguas, y no se analiza el comportamiento individual de cada parámetro. En la república del Paraguay las aguas superficiales deben cumplir con los estándares establecidos en la Clase II (MADES, 2006), en tal sentido los parámetros que se ajustan a la reglamentación son: $\mathrm{pH}$, turbidez y SDT. Por su parte los que estaban fuera del rango de los valores de referencia fueron: nitrato, NTK, PT, OD, $\mathrm{DBO}_{5}$ y coliformes fecales.

Los resultados del índice ICA se presentan en la Figura 2. El sitio ubicado en la cuenca alta (AG) fue el que presentó los valores más altos, con calificación "Buena" para ambos muestreos.
Posteriormente conforme se avanza en la cuenca del arroyo se observó un deterioro en su calidad con calificaciones "Regular" y "Mala”. En P1 la calidad bajó de Buena a Regular entre ambos muestreos. Tanto P2, P3 como P4 presentaron los valores más bajos y las peores calificaciones.

El índice IET (Figura 3), muestra que hubo un aumento general en el estado trófico en la segunda campaña de muestreo. Los valores más bajos de IET se registraron en los puntos P4 y P1 en la primera campaña (valores de IET entre 50,5 y 51,2 respectivamente). Los cambios más drásticos se produjeron en los puntos AG y $\mathrm{P} 4$, ya que en el primero pasó de un estado mesotrófico a supereutrófico (IET de 56,4 a 64,3), mientras que el segundo pasó del estado oligotrófico a supereutrófico (IET de 50,5 a 65,2).

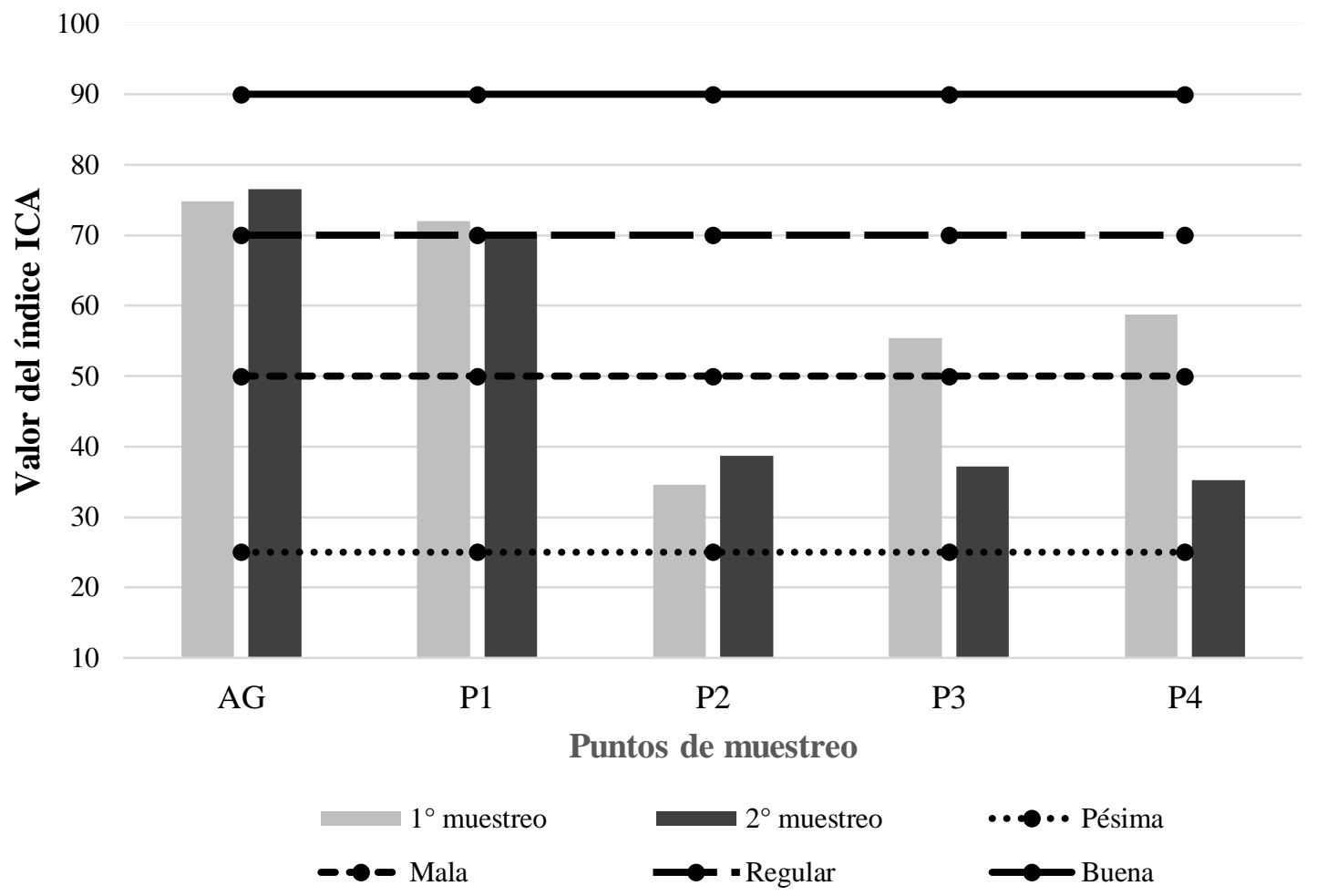

Figura 2. Índice de calidad de agua (ICA) por sitio y por campaña según el criterio de Brown et al. (1970). 


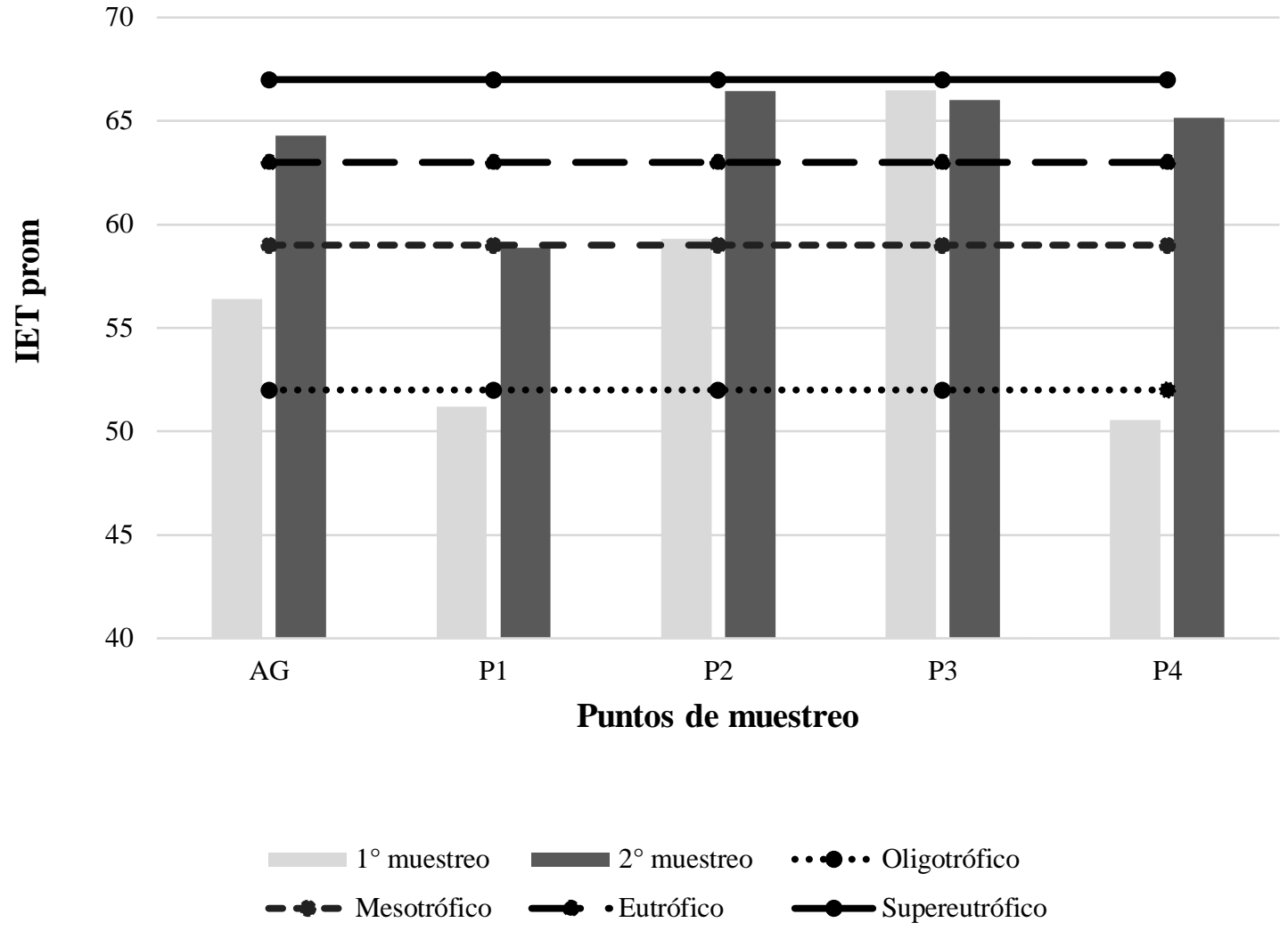

Figura 3. Índice de estado trófico (IET) por sitio y por campaña según el criterio de CETESB (2016) para ambientes lóticos.

En relación al zooplancton, fueron identificados 31 taxones, de los cuales todos fueron rotíferos (Tabla 2). Durante la primera campaña las especies más abundantes fueron Xenolepadella asticicola (AG), Proales reinhardti (P1), Lecane hamata (P2), Brachionus plicatillis, Lecane gillardi y Proales globulifera (P3). En la segunda campaña los rotíferos más dominantes fueron Ascomorpha ovalis (AG), Proales reinhardti (P1), Brachionus plicatillis (P2) y Ascomorpha ovalis (P3). En relación a la desembocadura del arroyo (P4) no se encontraron organismos zooplanctónicos, por ende, se registra una ausencia de diversidad.

Tabla 2. Especies registradas con sus densidades por localidad y por campaña.

\begin{tabular}{rlllllllllll}
\hline \multicolumn{1}{l}{} & \multicolumn{4}{c}{ Primera campaña } & \multicolumn{7}{c}{ Segunda Campaña } \\
Especies & AG & P1 & P2 & P3 & P4 & AG & P1 & P2 & P3 & P4 \\
\hline Ascomorpha ovalis & 0 & 0 & 100 & 100 & 0 & 1700 & 0 & 0 & 500 & 0 \\
Ascomorpha sp1 & 200 & 0 & 0 & 0 & 0 & 200 & 0 & 0 & 200 & 0 \\
Asplanchna sp1 & 200 & 0 & 0 & 0 & 0 & 0 & 0 & 0 & 0 & 0 \\
Asplanchna sp2 & 0 & 0 & 0 & 0 & 0 & 0 & 0 & 0 & 100 & 0 \\
\hline
\end{tabular}


Continúa Tabla 2

\begin{tabular}{|c|c|c|c|c|c|c|c|c|c|c|}
\hline \multirow[b]{2}{*}{ Especies } & \multicolumn{4}{|c|}{ Primera campaña } & \multicolumn{6}{|c|}{ Segunda Campaña } \\
\hline & $\mathbf{A G}$ & $\mathbf{P 1}$ & $\mathbf{P 2}$ & P3 & $\mathbf{P 4}$ & $\mathbf{A G}$ & P1 & $\mathbf{P 2}$ & P3 & $\mathbf{P 4}$ \\
\hline Brachionus calyciflorus & 0 & 0 & 0 & 0 & 0 & 100 & 0 & 100 & 0 & 0 \\
\hline Brachionus plicatillis & 200 & 0 & 0 & 300 & 0 & 400 & 0 & 120 & 0 & 0 \\
\hline & & & & & & & & 0 & & \\
\hline Brachionus sp. & 0 & 0 & 500 & 100 & 0 & 0 & 0 & 0 & 200 & 0 \\
\hline Cephalodella sp. & 200 & 0 & 0 & 0 & 0 & 0 & 800 & 100 & 100 & 0 \\
\hline Colurella obtusa & 200 & 0 & 0 & 0 & 0 & 0 & 0 & 0 & 0 & 0 \\
\hline Colurella sp1 & 0 & 0 & 200 & 0 & 0 & 400 & 0 & 500 & 200 & 0 \\
\hline Colurella tesselata & 0 & 0 & 200 & 100 & 0 & 0 & 0 & 0 & 0 & 0 \\
\hline Elosa worallii & 0 & 600 & 300 & 0 & 0 & 0 & 0 & 0 & 0 & 0 \\
\hline Euchlanis triqueta & 600 & 0 & 0 & 0 & 0 & 0 & 0 & 0 & 0 & 0 \\
\hline Filinia longiseta & 0 & 0 & 0 & 0 & 0 & 400 & 0 & 0 & 0 & 0 \\
\hline $\begin{array}{r}\text { Heterolepadella } \\
\text { heterodactyla }\end{array}$ & 100 & 0 & 0 & 0 & 0 & 0 & 0 & 0 & 0 & 0 \\
\hline Lecane gillardi & 500 & 0 & 100 & 300 & 0 & 0 & 0 & 200 & 0 & 0 \\
\hline Lecane hamata & 400 & 0 & 600 & 0 & 0 & 0 & 0 & 600 & 0 & 0 \\
\hline Lecane luna & 0 & 0 & 0 & 0 & 0 & 500 & 0 & 0 & 0 & 0 \\
\hline Lepadella ovalis & 100 & 0 & 300 & 200 & 0 & 600 & 0 & 0 & 300 & 0 \\
\hline Lepadella patella & 500 & 0 & 0 & 0 & 0 & 0 & 0 & 0 & 0 & 0 \\
\hline Philodina sp1 & 200 & 300 & 100 & 200 & 0 & 800 & 1600 & 600 & 0 & 0 \\
\hline Proales decipiens & 200 & 0 & 0 & 200 & 0 & 0 & 0 & 300 & 300 & 0 \\
\hline Proales globulifera & 0 & 0 & 0 & 300 & 0 & 0 & 0 & 300 & 0 & 0 \\
\hline Proales reinhardti & 0 & 1900 & 200 & 200 & 0 & 0 & 1900 & 0 & 0 & 0 \\
\hline Proales sp1 & 0 & 200 & 0 & 0 & 0 & 300 & 0 & 0 & 0 & 0 \\
\hline Proales theodora & 0 & 1000 & 100 & 0 & 0 & 300 & 0 & 300 & 0 & 0 \\
\hline Proalinopsis caudatus & 100 & 600 & 0 & 0 & 0 & 0 & 500 & 0 & 0 & 0 \\
\hline
\end{tabular}




\begin{tabular}{|c|c|c|c|c|c|c|c|c|c|c|}
\hline \multirow[b]{2}{*}{ Especies } & \multicolumn{5}{|c|}{ Primera campaña } & \multicolumn{5}{|c|}{ Segunda Campaña } \\
\hline & AG & P1 & $\mathbf{P 2}$ & $\mathbf{P 3}$ & P4 & $\mathbf{A G}$ & $\mathbf{P 1}$ & $\mathbf{P 2}$ & $\mathbf{P 3}$ & $\mathbf{P 4}$ \\
\hline Trichocerca pusilla & 0 & 0 & 0 & 0 & 0 & 1400 & 0 & 0 & 0 & 0 \\
\hline Trichocerca sp. & 0 & 0 & 0 & 0 & 0 & 0 & 200 & 0 & 0 & 0 \\
\hline Trichocerca sulcata & 300 & 0 & 0 & 0 & 0 & 500 & 0 & 0 & 200 & 0 \\
\hline Xenolepadella asticicola & 900 & 200 & 100 & 0 & 0 & 0 & 0 & 300 & 0 & 0 \\
\hline
\end{tabular}

Se observó una disminución en la riqueza de taxones en la segunda campaña en todos los puntos (Tabla 3), en donde la naciente AG presentó los mayores valores en ambas campañas (16 y 13 taxones respectivamente). Mientras que los brazos P1, P2 y P3, los valores bajaron entre 7 a 12 taxones en el primer muestreo y entre 5 a 11 taxones en el segundo muestreo. La equidad de la comunidad zooplanctónica también presentó un patrón similar de descenso en el segundo muestreo, excepto en el punto P1 donde hubo un leve ascenso de la equidad $(0,85$ y 0,86 en el primer y segundo muestreo respectivamente). Los puntos de mayor equidad fueron en el brazo P3 en ambas campañas (0,97 y 0,95 en el primer y segundo muestreo respectivamente).
Con los ensayos de toxicidad aguda con Daphnia magna y Lactuca sativa, solo se detectaron efectos letales para P4 en el segundo muestreo. Se produjo mortalidad de todos los individuos expuestos a la muestra al $100 \%$, no detectando toxicidad con las diluciones, motivo por el cual no se pudo determinar la $\mathrm{CL}_{50}$ para esta muestra. En general, fuera de esta muestra de P4, los demás ensayos no arrojaron efectos tóxicos agudos detectables con los bioindicadores utilizados para las muestras de aguas, esto para el parámetro de punto final seleccionado $\left(\mathrm{CE}_{50} / \mathrm{CL}_{50}\right)$. Los valores de IP en $L$. sativa tendieron a ser levemente positivos, indicando un estímulo, antes que una inhibición del crecimiento radicular.

Tabla 3. Biodiversidad del zooplancton en las dos campañas.

\begin{tabular}{lllllllllll}
\hline Local & AG & & P1 & & P2 & & P3 & \multicolumn{2}{c}{ P4 } \\
Diversidad & S & P & S & P & S & P & S & P & S & P \\
\hline $\mathbf{I}^{\circ}$ muestreo & 16 & 0,92 & 7 & 0,85 & 12 & 0,92 & 10 & 0.97 & 0 & 0 \\
$\mathbf{2}^{\circ}$ muestreo & 13 & 0,90 & 5 & 0,86 & 11 & 0,90 & 9 & 0,95 & 0 & 0 \\
\hline
\end{tabular}




\section{DISCUSIÓN}

Los valores de los parámetros fisicoquímicos $\mathrm{pH}$, turbidez y SDT se mantuvieron dentro del rango establecido para aguas superficiales de Clase II. Los mismos, son similares a los observados en el estudio del Arroyo Caañabe, donde los parámetros que presentaron condiciones óptimas fueron: $\mathrm{pH}$, turbidez, nitratos, sulfatos, dureza, OD, STD, y metales tales como cadmio, cromo y plomo (López Arias et al., 2016). Adicionalmente, la cuenca baja del Arroyo Guazú, correspondientes a los puntos P3 y P4, mostraron concentraciones de 16,8-39,5 $\mathrm{mgO}_{2}$.mg. L $\mathrm{L}^{-1}\left(\mathrm{DBO}_{5}\right), 64-241$ (DQO mg. L $\left.{ }^{-1}\right), 0,1-$ 1,05 (NTK), y 0,22-1,48 mg. $\mathrm{L}^{-1}$ (PT) como se muestran en la Tabla 1. Estos valores son similares a los reportados por Silvero \& Kunze (2013) en el informe sobre las actividades humanas que condicionan la calidad del agua del Río Paraguay, Lago Ypacaraí y otros afluentes. En el informe, se citan concentraciones de parámetros fisicoquímicos de arroyos urbanos de Gran Asunción, entre estos los valores para $\mathrm{DBO}_{5}$, DQO, NTK, PT en mg.L $\mathrm{L}^{-1}$ respectivamente son: 6, 51, 11,8 y 1,66 (Arroyo Ytay, zona Aeropuerto); 100, 165, 24,9 y 8,9 (Arroyo Mburicao, zona Tablada Nueva) y 12, 36, 8,46 y 0,67 para el Arroyo Lambaré.

Por otro lado, los resultados de los análisis de $\mathrm{CF}$ indicaron que las muestras de agua poseen cargas microbianas inferiores al nivel máximo permitido de 1.000 ufc.100 $\mathrm{mL}^{-1}$ (Tabla 1), a excepción de la muestra del sitio P2 que en el primer muestreo presentó valores de CF de 1.117 ufc. $100 \mathrm{~mL}^{-1}$. De esta manera, desde un aspecto microbiológico se pueden asignar al Arroyo Guazú calificaciones entre Clase II (AG, P1, P3 y P4) y
Clase III (para P2). Esta clasificación es similar a los obtenido en un estudio de calidad de agua del Arroyo San Lorenzo de la Ciudad de Pilar, donde se reportaron valores de: $\mathrm{OD}, \mathrm{DBO}_{5}$, turbidez, niveles de la bacteria E. coli 3.700- 5.800 ufc. $100 \mathrm{~mL}^{-1}$, y que permitieron clasificar sectores de la cuenca como aguas de Clase II y Clase III (Olmedo \& Fossati, 2019). De igual modo, López Arias et al., (2016) evaluaron las aguas del Arroyo Caañabe, obteniendo valores de CF 1000 ufc. $100 \mathrm{~mL}^{-1}$ semejantes a los obtenidos en esta investigación.

Considerando que las concentraciones de seis de los parámetros analizados no cumplen con los estándares para aguas de Clase II, en por lo menos un muestreo por sitio (nitratos, NTK, PT, OD, $\mathrm{DBO}_{5}$ y $\mathrm{CF}$ ), se clasifica a las aguas del Arroyo Guazú como de Clase III. Según el MADES (2002), estas aguas solo pueden ser destinadas para el abastecimiento doméstico después de un tratamiento especial, irrigación arbórea, de jardín, forrajeras y para recreación de contacto secundario.

De esta manera el deterioro de la calidad de este cause imposibilita el uso que se les otorga a aguas de Clase II para las siguientes actividades: el abastecimiento doméstico después de los tratamientos convencionales, la protección de las comunidades acuáticas, para recreación de contacto primario (natación), irrigación de hortalizas que son consumidas crudas, ni para la cría natural y/o intensiva (acuicultura) de especies destinadas para la alimentación humana.

Los resultados del ICA indican que la cuenca baja del Arroyo Guazú posee los niveles más bajos de calidad con calificaciones Mala y Regular. Las aguas con categoría "Regular" y "Mala" tienen generalmente menos diversidad de organismos acuáticos como consecuencia de la presión 
ambiental generada por la contaminación (Servicio Nacional de Estudios Territoriales, 2012). A su vez, los índices IET reflejan contaminación por nutrientes, principalmente por fosfatos, lo cual deriva a una disminución en la calidad de agua (Orozco Barrenetxea, 2003). En este trabajo, la ausencia de grupos relevantes para la biodiversidad como los microcrustáceos, es decir, cladóceros y copépodos (Conde-Porcuna et al., 2004), indican una perturbación ambiental generada por la mala calidad de las aguas del Arroyo Guazú, ya que los mencionados invertebrados son generalmente sensibles a la contaminación, mientras que, algunas especies de rotíferos como las del género Brachionus o Proales (encontrados en los puntos con mayor IET) suelen ser considerados como bioindicadores de eutrofización (Parmar et al., 2016). La disminución de la biodiversidad en términos de riqueza y uniformidad puede afectar algunos servicios ecosistémicos tales como, la calidad de agua, el paisaje y la producción primaria y secundaria acuática (Aylward et al., 2005; Duffy et al., 2007).

En lo que respecta a los ensayos ecotoxicológicos, las muestras de aguas del Arroyo Guazú no presentaron efectos tóxicos agudos medibles como $\mathrm{CL}_{50}$ o $\mathrm{CE}_{50}$, tanto en $D$. magna y $L$. sativa. Los bajos niveles de toxicidad aguda pueden ser explicados por la ausencia o bajas concentraciones de agentes recalcitrantes que provocan efectos letales e inhibitorios Otros estudios también han reportado escasos efectos adversos detectables utilizando diferentes organismos como T. wisconsinensis, D. magna, y D. rerio (López Arias et al., 2016); y en los arroyos Pirayú y Yukyry (López et al., 2013) teniendo en cuenta los parámetros $\mathrm{CE}_{50} / \mathrm{CL}_{50}$ utilizados en este estudio.

Los parámetros y los índices de calidad obtenidos en este estudio indican que las aguas del Arroyo Guazú presentan una baja calidad debido a la contaminación severa principalmente desde el punto de vista fisicoquímico. Esta situación de deterioro estaría estrechamente vinculado a tres grandes problemáticas: (1) el vertido de residuos sólidos al arroyo que se observa a lo largo de todo el cauce, asociado a prácticas socioculturales y la baja cobertura de recolección que solo llega al $22,9 \%$ de la Ciudad de San Antonio y del $30 \%$ para la Ciudad de Nemby. (2) La cobertura de alcantarillado sanitario que apenas abarca al 6,5\% en la Ciudad de San Antonio (Empresa de Servicios Sanitarios del Paraguay, 2015). Y finalmente, (3) la actividad comercial e industrial de la zona, entre las que destacan las curtiembres que permanentemente son intervenidas por denuncia de los pobladores (Ruiz Díaz, 2020). Tales impactos antrópicos están afectando la biodiversidad acuática y generan un grave problema a nivel ecológico, sanitario y económico al imposibilitar el uso de esta cause hídrico como agua superficial. Por lo tanto, se recomienda un monitoreo constante en esta cuenca considerando que sus aguas desembocan directamente al Río Paraguay, así como la implementación de medidas paliativas (regulación de la descarga de contaminantes o implementación de técnicas de biorremediación en su ribera).

Declaración de interés. Los autores declaran no tener conflicto de intereses. 


\section{REFERENCIAS BIBLIOGRÁFICAS}

Alonso, F. \& López, T. (2015). Efectos tóxicos agudos de metales pesados sobre el crecimiento radicular de. Reportes Científicos de LA FACEN, 6(1), 21-29. http://www.facen.una.py/wpcontent/uploads/2015/03/revista nro6 1.pdf

APHA/AWWA/WEF. (2012). Standard Methods for the Examination of Water and Wastewater. Standard Methods. 22 ed. Washington. American Public Health Association.

Aylward, B., Bandyopadhyay, J. \& Belausteguigotia, J. C. (2005). Freshwater Ecosystem Services. In Ecosystems and Human Well-being: Policy responses. Millennium Ecosystem Assessment Board (pp. 213-255).

Brown, R., Mcclelland, N., Deininger, R. \& Tozer, R. (1970). A Water Quality Index-Do we Dare? Water and Sewage Works, 11, 339-343.

Carlson, R. E. (1977). A trophic state index for lakes1. Limnology and Oceanography, 22(2), 361-369. https://doi.org/10.4319/1o.1977.22.2.0361

Castillo Morales, G. (2004). Ensayos toxicológicos y métodos de evaluación de calidad de aguas (1ra ed.). México. IMTA.

Companhia Ambiental do Estado de São Paulo. (2016). Índices de Qualidade das Águas. Qualidade Das Águas Interiores No Estado de São Paulo. CETESB. https://cetesb.sp.gov.br/aguas-interiores/wpcontent/uploads/sites/12/2018/03/Ap\%C3\%AAndice-D$\% \mathrm{C} 3 \% 8 \mathrm{Dndices}-\mathrm{de}-Q u a l i d a d e-d a s-\% \mathrm{C} 3 \% 81$ guas-1.pdf

Conde-Porcuna J., Ramos-Rodríguez, E. \& Morales-Banquero, R. (2004). El zooplancton como integrante de la estructura trófica de los ecosistemas. Ecosistemas, 13(2), 23-29.

Craggs, A. (2017). Organización Mundial de la Salud. Enfermedades transmitidas por el agua.https://w ww.who.int/water_sanitation_health/diseasesrisks/diseases/es/

Duffy, J. E., Bradley, J., France, K. E. \& Mcintyre, P. B. (2007). The functional role of biodiversity in ecosystems : incorporating trophic complexity. Ecology Letters, 10, 522-538. https://doi.org/10.1111/j.14610248.2007.01037.x

Elmoor-Loureiro, L. (1997). Manual de identificação de cladóceros Limnicos do Brasil. Brasilia. Universa.

Empresa de Servicios Sanitarios del Paraguay (2015). Proyecto de Adecuación Ambiental del sistema de alcantarillado sanitario de la Ciudad de San Antonio. http://mades.gov.py/sites/default/files/users/control/essa p_san.antonio_r.grommeck.pdf

Enciso, C., Brunelli, M. L., Colmán, F., Silvero, J., Almada, M. \& Cruzans, G. (2012). Cuenca Hídrica del Arroyo San Lorenzo , Departamento Central Informe técnico Manejo Sostenible y Protección de las Aguas Subterráneas en Paraguay Cuenca Hídrica del Arroyo San Lorenzo , Departamento Central. http://www.mades.gov.py/wpcontent/uploads/2018/06/Informe-Final-San-Lorenzo.pdf

Espino G., Hernandez S. \& Carbajal J. (2000). Organismos indicadores de la calidad del agua y de la contaminación (bioindicadores). México. Plaza y Valdéz S.A. 551 pp.

FAO. (2015). AQUASTAT Perfil de País - Paraguay. Roma, Italia. Organización de las Naciones Unidas para la Alimentación y la Agricultura.
Hodkinson, I. D. \& Jackson, J. K. (2005). Terrestrial and aquatic invertebrates as bioindicators for environmental monitoring, with particular reference to mountain ecosystems. Environmental Management, 35(5), 649666. https://doi.org/10.1007/s00267-004-0211-x

Houben, G., Rojas, C., Romero, A., Cabral, N., Olavarrieta, A., INCLAM, S. (2017). Estudio de recursos hídricos y vulnerabilidad climática del Acuífero Patiño: Diagnóstico de Acuífero Patiño. Asunción. http://opengeo.pol.una.py/descargas/Repositorio_estudio s_AP/PR-T1207-Diagnostico.pdf

Joko, C. Y. (2011). Taxonomía de rotíferos monogonontas da planície de inundação do alto rio Paraná ( $M S$ / PR ). (Tesis doctoral). Universidade Estadual de Maringá, Brasil.

Londoño Franco, L. F., Londoño Muñoz, P. T. \& Muñoz Garcia, F. G. (2016). Los riesgos de los metales pesados en la salud humana y animal. Biotecnología en el sector agropecuario y agroindustrial, 14(2), 145-153. https://doi.org/10.18684/bsaa(14)145-153

López Arias, T. R., Fernández Peralta, V. F., Franco de Diana, D. M., Galeano Delgado, E. F., Alonso Márquez, F. S., Benítez Martínez, M. D., López Vera, M. E., Ayala, H. I., Bobadilla Giménez, N., Benítez Resquín, L. M. \& Mazó Bareiro, C. G. (2016). Índices de calidad ambiental de aguas del Arroyo Caañabe mediante tests microbiológicos y ecotoxicológico. Ambiente e Agua An Interdisciplinary Journal of Applied Science, 11(3), 548. https://doi.org/10.4136/ambi-agua.1703

López, T., Franco de Diana, D., Fernández Peralta, V., Torres, C., Benítez, M., Ramond, F., López Lovera, M. E., Giménez, N. B., Kurita, G., Acuña, R., Caballero, R. \& López, D. (2013). Diagnóstico ecotoxicológico y genotóxico de los afluentes del lago Ypacarai, mediante bioensayos con Daphnia magna Straus, Danio rerio , Lactuca sativa L y Allium cepa L. Asunción. FACEN, UNA. http://sdi.cnc.una.py/catbib/documentos/647.pdf

Magurran, A. E. (2005). Measuring biological diversity. Environmental and Ecological Statistics. Blackwell Science Ltd.

Ministerio del Ambiente y Desarrollo Sostenible. (2002). Resolución $N^{\circ} 222 / 02$ "Por la cual se establece el padrón de calidad de las aguas en el territorio nacional". Asunción. MADES

Ministerio del Ambiente y Desarrollo Sostenible. (2006). Resolución $N^{\circ}$ 255/06 "Por la cual se establece la clasificación de las aguas superficiales de la República del Paraguay". Asunción, Paraguay.

Monte Domecq, R., \& Báez, J., (2007). Balance hídrico integrado del Acuífero Patiño. Asunción-Paraguay: Servicio Nacional de Saneamiento Ambiental SENASA Paraguay.https://www.geologiadelparaguay.com.py/Bal ance-Hidrico-Acuifero-Patinho.pdf

Olmedo, D. \& Fossati, N. (2019). Evaluación preliminar de la calidad del agua de un arroyo urbano en la ciudad de Pilar: Cuenca baja del arroyo San Lorenzo. Jetypeka, Revista Científica Multidisciplinaria, 1(1), 90-104. https://jetypeka.unp.edu.py/index.php/jtpk/article/view/1 6

Orozco Barrenetxea, C., Pérez Serrano, A., González Delgado, M. N., Rodríguez Vidal, F. J. \& Alfayate Blanco, J. M. (2003). Contaminación Ambiental. Una visión desde la química. Internacional Thompson Editores. Madrid. Paraninfo. $281 \mathrm{p}$.

Otero, A. R. (2019). Paraguay Medioambiente. Asuncenos desafían la prohibición de bañarse en aguas contaminadas del río Paraguay. Agencia EFE. 
https://www.efe.com/efe/america/cronicas/asuncenosdesafian-la-prohibicion-de-banarse-enaguascontaminadas-del-rio-paraguay/50000490-3867584

Parmar, T. K., Rawtani, D. \& Agrawal, Y. K. (2016). Bioindicators: the natural indicator of environmental pollution. Frontiers in Life Science, 9(2), 110-118. https://doi.org/10.1080/21553769.2016.1162753

QGIS. (2020). Sistema de información geográfica QGIS. Proyecto de la Fundación Geoespacial de Código Abierto. https://qgis.org/es/site/

Ruiz Díaz, H. (2020). Persiste contaminación del Arroyo Guazú en San Antonio. https://www.abc.com.py/nacionales/2020/09/19/persistecontaminacion-del-arroyo-guasu-en-san-antonio/

Ruiz Díaz, H. R. (2019). Arroyo Guasu de San Antonio se tiñó de rojo - Nacionales. ABC Color. https://www.abc.com.py/nacionales/2019/12/09/arroyoguasu-de-san-antonio-se-tino-de-rojo/
Samarez Valer I., Rimarachín V., Palma C., Arana J., Ortega H., Correa V. \& Hidalgo del Águila M. (2014). Métodos de colecta, Identificación y Análisis de Comunidades Biológicas: Plancton, Perifiton, Bentos (Macroinvertebrados) y Necton (Peces) en Aguas Continentales del Perú. Universidad Nacional Mayor de San Marcos.

Servicio Nacional de Estudios Territoriales. (2012). Índice de Calidad de Agua

ICA.SNET.http://www.snet.gob.sv/Hidrologia/Documen tos/calculoICA.pdf

Silvero, J. \& Kunze, F. (2013). Informaciones sobre las actividades humanas que condicionan la calidad del agua del Rio Paraguay, Lago Ypacarai y otros afluentes. Asunción. Secretaría del Ambiente. 